\title{
LIMIT CYCLES IN A KOLMOGOROV-TYPE MODEL
}

\author{
XUN-CHENG HUANG \\ Department of Mathematics \\ New Jersey Institute of Technology \\ Newark, New Jersey 07102 U.S.A. \\ (Received June 2, 1989 and in revised form February 2, 1990)
}

\begin{abstract}
In this paper, a Kolmogorov-type model, which includes the Gause-type model (Kuang and Freedman, 1988), the general predator-prey model (Huang 1988, Huang and Merrill 1989), and many other specialized models, is studied. The stability of equilibrium points, the existence and uniqueness of limit cycles in the model are proved.
\end{abstract}

KEY WORDS AND PHRASES. Kolmogorov model, predator-prey model, limit cycles. 1980 AMS SUBJECT CLASSIFICATION CODE. 92A15.

\section{INTRODUCTION}

The topic of limit cycles is interesting both in mathematics and in science. This concept first appeared in print in the famous paper by Poincare $(1881,1882,1885,1886)$. Then in 1926, van der Pol proposed an equation in the study of a self-sustained oscillation occurring in a vacuum tube circuit which showed that the closed orbit in the phase plane of the equation is a limit cycle as considered by Poincare. After this observation, the existence, non-existence, uniqueness and other properties of limit cycles were studied extensively by mathematicians and physicists.

By the 1950's, many models from physics, engineering, chemistry, biology, economics, etc. were displayed as plane autonomous systems with limit cycles. Since then, more and more mathematicians and scientists have been attracted to the topic. Even in the renowned 23 Hilbert problems, you will find a place for limit cycles, specifically in the 16th problem (see [9] for example).

In mathematical modeling of ecological systems, since the papers of May (1972[8]), and Albrecht, Gatzke and Wax (1973[1]) finding conditions that guarantee the uniqueness of a limit cycle in predator-prey model has been considered an outstanding problem. Recently, several results have been published (see, e.g. Cheng 1981[2], Kuang and Freedman 1988[7], Huang 1988[5], Huang and Merrill 1989[6]).

In this paper, a general model of Kolmogorov-type is investigated. This model takes into account all of the above models as special cases. We are going to prove the stability of the equilibrium points, the existence and the uniqueness conditions of limit cycles. Several known theorems will be easily derived again as an illustration of our theorem.

The method used in this paper can be employed for use in the study of general Kolmogorov systems and will be published elsewhere for further study. 


\section{THE MODEL.}

Consider the model

$$
\begin{aligned}
& \frac{d x}{d t}=\phi(x)(F(x)-\pi(y)) \\
& \frac{d y}{d t}=\varrho(y)(\psi(x)+\xi(y))
\end{aligned}
$$

where $x$ is the prey density, $y$ is the predator density, $\phi(x) F(x)$ is the intrinsic growth rate of the prey in the absence of predators, and $\varrho(y) \xi(y)$ is the intrinsic rate of the increasing (or decreasing) of the predator. The term $\phi(x) \pi(y)$ represents the functional response of the predator, i.e.

$$
\frac{\phi(x) \pi(y)}{x}
$$

is the rate of prey consumption per predator. Most of the authors simply take $\pi(y)=y$, but a function $\pi(y)$ that increases slower than the linear could be used to model interference among predators with each other's hunting, or faster than the linear could be used to model predator cooperation [3]. The term $\varrho(y) \psi(x)$ is the response of the predator, which means the difference of the actual rate of increase and the intrinsic rate of increase of the predator. In particular,

$$
\frac{\varrho(y) \psi(0)+\xi(y)}{y}
$$

is the death rate of the predator in the absence of prey.

For the following discussion, we need to assume:

$\left(\mathrm{H}_{1}\right): \phi, \psi, \pi, \varrho, \xi \in C^{1}([0, \infty)) ; F \in C^{1}(0, \infty), F(0) \in(0, \infty] \phi(0)=\pi(0)=$ $\varrho(0)=\xi(0)=0, \phi^{\prime}>0$ for $x \geq 0, \pi^{\prime}>0, \varrho^{\prime}>0, \xi^{\prime} \leq 0$ for $y \geq 0$; there exists $\bar{x}>0$ such that $\psi(\bar{x})=0, \psi^{\prime}(x)>0$ for $x \neq \bar{x}$. Moreover, $\phi(x)$ is bounded by some linear function for $0 \leq x \leq K .\left(\mathrm{H}_{1}^{*}\right)$

$\left(\mathrm{H}_{2}\right)$ : The curve $\pi(y)-F(x)=0$ is defined for all $x>0$, and $\psi(x)+\xi(y)=0$ is defined for $0<x \leq K$.

$\left(\mathrm{H}_{3}\right)$ : There exists $K>\bar{x}$ such that $F(K)=0, F^{\prime}(K)<0, F(x)>0$ for all $0<x<K$, and for any $\bar{k}>K, F^{\prime}(\bar{k}) \neq 0$ if $\mathbf{F}(\bar{k})=0$.

Moreover, these exists a $K^{*}<\infty$ such that $F\left(K^{*}\right)=0$ and $F(x) \neq 0$ for any $x>K^{*}$.

$\left(\mathrm{H}_{4}\right)$ : There exist positive numbers $M$ and $\epsilon_{M}$ such that $\pi(y) \geq M \varrho(y)$ for $y \geq \epsilon_{M}$, and also there exist positive $N$ and $\epsilon_{N}$ such that $\varrho(y) \geq N y$ for $y \geq \epsilon_{N}$.

The constant $K$ in $\left(\mathrm{H}_{1}\right)$ and $\left(\mathrm{H}_{2}\right)$ is the same as in $\left(\mathrm{H}_{3}\right)$.

It is possible to have $F(0)=\infty$ in most of this discussion. In that case $(0,0)$ is no longer an equilibrium point. This discussion is in the interior of the first quadrant.

Clearly, system (2.1) consists of those studied by Lotka and Volterra, Gause, Rosenzwieg and MacArthur (1963), Generalized Gause (Freedman, 1980), Hsu (1978), Hsu, Hubbell and Waltman (1978), Kazarinnoff and Driessche (1978), Cheng (1981), Liou and Cheng (1988), Kuang and Freedman (1988), Huang (1988), Huang and Merrill (1989). For example, Kuang and Freedman model (1988) is a special case of system (2.1) with $F(0)<$ $\infty, F(x)<0$ for all $x>K$, and $\xi(y)=0$. Also, the assumptions $\left(\mathrm{H}_{1}^{*}\right),\left(\mathrm{H}_{2}\right)$ and $\left(\mathrm{H}_{4}\right)$ employed here but not employed in Kuang and Freedman we believe should be required there, also. 


\section{THEOREMS AND PROOFS.}

Clearly, system (2.1) with assumptions $\left(\mathrm{H}_{1}\right)-\left(\mathrm{H}_{4}\right)$ has a positive equilibrium $\left(x^{*}, y^{*}\right)$, where $\bar{x} \leq x^{*}<K$ and one or more saddles, for example, $(K, 0)$, and $(0,0)$ (if $F(0)<\infty)$.

For the stability of $\left(x^{*}, y^{*}\right)$ we have:

THEOREM 3.1. Let

$$
H(x, y)=\phi(x) F^{\prime}(x)+\varrho(y) \xi^{\prime}(y) .
$$

$H\left(x^{*}, y^{*}\right)<0$, the equilibrium $\left(x^{*}, y^{*}\right)$ is stable, while $H\left(x^{*}, y^{*}\right)>0$ unstable.

PROOF. The Jacobian of system $(2.1)$ at $\left(x^{*}, y^{*}\right)$ is

$$
J\left(x^{*}, y^{*}\right)=\left(\begin{array}{rr}
\phi\left(x^{*}\right) F^{\prime}\left(x^{*}\right), & -\phi\left(x^{*}\right) \pi^{\prime}\left(y^{*}\right) \\
\varrho\left(y^{*}\right) \psi^{\prime}\left(x^{*}\right), & \varrho\left(y^{*}\right) \xi^{\prime}\left(y^{*}\right)
\end{array}\right)
$$

and the signs of the real parts of the eigenvalues are determined by

$$
H\left(x^{*}, y^{*}\right)=\phi\left(x^{*}\right) F^{\prime}\left(x^{*}\right)+\varrho\left(y^{*}\right) \xi^{\prime}\left(y^{*}\right) .
$$

It is easy to see that Theorem 3.1 is valid.

\section{THEOREM 3.2. Suppose}

(i) there exists a $c_{0} \geq 1$ such that $M / c_{0} \phi^{\prime}(x)-\psi^{\prime}(x) \leq 0$ for all $\mathrm{x} \geq 0$ and

$$
\lim _{x \rightarrow \infty}\left(\frac{M}{c_{0}} \phi(x)-\psi(x)\right)=C_{\infty}>0
$$

(ii) $\quad F(x)<0$ for $x>K^{*}$, where $K^{*}$ is as in $\left(\mathrm{H}_{3}\right)$.

Let $x(t), y(t)$ be the solution of system (2.1) with a positive initial condition $x(0)=x_{0}>0, y(0)=y_{0}>0$. Then there exists $T>0$ such that $0<x(t)<T$ and $0<y(t)<T$ for all $t \geq 0$, and exists $t_{0} \geq 0$ such that $0<x(t)<K$ for all $t \geq t_{0}$

PROOF. By the phase portrait analysis, it is clear that $x(t), y(t)>0$ for all $t \geq 0$. If $x(0)<K$, then $x(t)<K$ for all $t \geq 0$. Otherwise, there exists $t_{1}>0$ such that

$$
x\left(t_{1}\right)=K \text { and } x^{\prime}\left(t_{1}\right) \geq 0 .
$$

But (2.1) implies that

$$
x^{\prime}\left(t_{1}\right)=-\phi(K) \pi(K)<0
$$

which is a contradiction.

If $\left(x_{0}, y_{0}\right) \in \Omega_{1}=\{(x, y): \psi(x)+\xi(y) \geq 0, F(x)-\pi(y)<0, y>0\}$, then (2.1) and $\left(\mathrm{H}_{2}\right)$ imply that either $x(t)$ decreases to some constant, or there exists $t_{2}>0$ such that $x\left(t_{2}\right)<K$. By the same argument as the case $x(0)<K$, it is necessary that

$$
x(t)<K \text { for all } t \geq t_{2}
$$

Hence, for all $t \geq 0$,

$$
x(t) \leq T_{1}=\max \{K, x(0)\} .
$$

If $\left(x_{0}, y_{0}\right) \in \Omega_{2}=\{(x, y): \psi(x)+\xi(y) \geq 0, F(x)-\pi(y)>0, y>0\}$, by the phase portrait analysis, the trajectory starting at $\left(x_{0}, y_{0}\right)$ will cross the boundary of $\Omega_{2}$ into $\Omega_{1}$. Hence there exists $t_{3}>0$, such that

$$
x(t)<K \text { for all } t \geq t_{3},
$$

and

$$
x(t)<K^{*} \text { for all } t \geq 0 \text {. }
$$

Therefore, $x(t)$ is bounded. 
To show $y(t)$ is bounded, by using $\left(\mathrm{H}_{4}\right)$, we estimate the following

$$
\begin{aligned}
\frac{1}{c_{0}} \frac{d x}{d t}+\frac{d y}{d t} & =\frac{1}{c_{0}} \phi(x)(F(x)-\pi(y))+\varrho(y)(\psi(x)+\xi(y)) \\
& \leq \frac{1}{c_{0}} \phi(x) F(x)-\varrho(y)\left(\frac{M}{c_{0}} \phi(x)-\psi(x)\right) \\
& \leq \frac{1}{c_{0}} \phi(x) F(x)-\varrho(y) C_{\infty} \\
& =\frac{1}{c_{0}}\left(\phi(x) F(x)+C_{\infty} N x(t)\right)-C_{\infty}(\varrho(y)-N y(t)) \\
& \leq \frac{1}{c_{0}}\left(\phi(x) F(x)+C_{\infty} N x(t)\right)-C_{\infty} N\left(\frac{1}{c_{0}} x(t)+y(t)\right) \\
& =M_{0}-N_{0}\left(\frac{1}{c_{0}} x(t)+y(t)\right),
\end{aligned}
$$

where $M_{0}=\max \left(\frac{1}{c_{0}} \phi(x) F(x)+\frac{C_{\infty}}{c_{0}} N x(t)\right), \quad N_{0}=C_{\infty} N$

Since $x(t)$ is bounded, so are $\phi(x)$ and $F(x)$. Thus $M_{0}$ is a constant.

Now, let $z(t)$ satisfy

$$
\begin{aligned}
& \frac{d z}{d t}=M_{0}-N_{0} z(t) \\
& z(0)=\frac{1}{c_{0}} x(0)+y(0)
\end{aligned}
$$

Then

$$
z(t)=z(0) e^{-N_{0} t}+\frac{M_{0}}{N_{0}}\left(1-e^{-N_{0} t}\right)
$$

Since $z(t) \geq \frac{1}{c_{0}} x(t)+y(t)>0$ for all $t \geq 0$ and $z(t), x(t)$ are bounded, $y(t)$ is bounded. Let $t_{0}=\max \left\{t_{1}, t_{2}\right\}$, then $0<x(t)<K$ for all $t \geq t_{0}$.

The proof of Theorem 3.2 is completed.

From the proof of Theorem 3.2, we have

THEOREM 3.3. Under the assumptions as in Theorem 3.2, if $\left(x^{*}, y^{*}\right)$ is stable, it is asymptotically global stable.

In the case when $\left(x^{*}, y^{*}\right)$ is unstable, we have

THEOREM 3.4. There exists at least one limit cycle around $\left(x^{*}, y^{*}\right)$ if $\left(x^{*}, y^{*}\right)$ is a unstable equilibrium point of system (2.1)

PROOF. Let $\ell_{1}$ be the curve $\psi(x)+\xi(y)=0$. If $\ell_{1}$ intersects the ray $x=K, y>0$ at $P_{1}\left(x_{p_{1}}, y_{p_{1}}\right)$, then

$$
\Gamma_{1}=\overline{A P_{1}} \cup \overline{P_{1} B} \cup \overline{B O} \cup \overline{O A},
$$

where $A=\left(x_{p_{1}}, 0\right), B=\left(0, y_{p_{1}}\right), O=(0,0)$, is the boundary and any trajectory which intersects it either crosses from exterior to interior or remains on it. Therefore, by the Poincare-Bendixson annular region theorem, there exists at least one limit cycle around $\left(x^{*}, y^{*}\right)$.

If $\ell_{1}$ does not intersect the ray $x=K, y>0$ at all, letting

$$
m_{0}=\max _{x \leq x<K}\{F(x)\}>0,
$$

then by $\left(\mathrm{H}_{2}\right)$, there exists $y_{1}$ such that

$$
\pi\left(y_{1}\right)=m_{0} .
$$


Consider the auxiliary system

$$
\begin{aligned}
& \frac{d x}{d t}=\phi(x)\left(m_{0}-\pi(y)\right) \\
& \frac{d y}{d t}=\varrho(y)\left(\psi(K)+\xi\left(2 y_{1}\right)\right)
\end{aligned}
$$

and the trajectory starting at point $P_{1}^{\prime}\left(K, 2 y_{1}\right)$ will intersect the curve $\ell_{1}$ since $\left(\mathrm{H}_{1}^{*}\right)$.

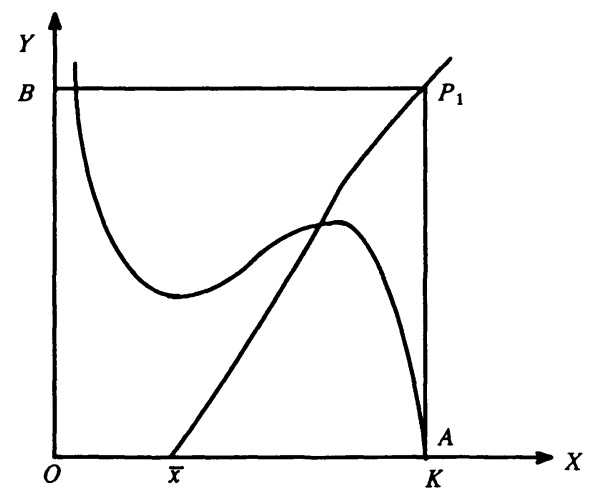

Fig. $1 \quad \Gamma_{1}=\overline{A P_{1}} \cup \overline{P_{1} B} \cup \overline{B O} \cup \overline{O A} \quad$ is the boundary of the Poincare-Bendixson annular region.

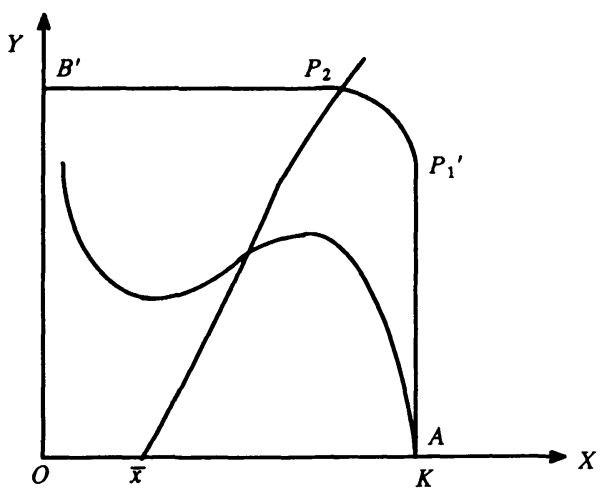

Fig. 2 If $\ell_{1}$ does not intersect the ray $\mathrm{x}=\mathrm{K}$, y $>0, \quad \Gamma_{2}=\overline{A P_{1}^{\prime}} \cup \overparen{P_{1}{ }^{\prime} P_{2}} \cup \overline{P_{2} \bar{B}^{\prime}} \cup \overline{B^{\prime} O} \cup \overline{O A}$ is the boundary of the Poincare-Bendixson annular region.

Suppose the intersection is $P_{2}\left(x_{p_{2}}, y_{p_{2}}\right)$. Let $B^{\prime}=\left(0, y_{p_{2}}\right)$. Then

$$
\Gamma_{2}=\overline{A P_{1}^{\prime}} \cup \overparen{P_{1}^{\prime} P_{2}} \cup \overline{P_{2} B^{\prime}} \cup \overline{B^{\prime} O} \cup \overline{O A}
$$

is a boundary of an annular region. Since

$$
\begin{aligned}
& F(x)-\pi(y) \leq m_{0}-\pi(y)<0 \\
& \psi(x)+\xi(y) \leq \psi(K)+\xi\left(2 y_{1}\right),
\end{aligned}
$$

any trajectory intersects $\Gamma_{2}$ will either cross from exterior to interior or remain on it. The Poincare-Bendixson Theorem guarantees that there is at least one limit cycle inside $\Gamma_{2}$.

Therefore, in any cases there exists at least one limit cycle around $\left(x^{*}, y^{*}\right)$.

Now, for the proving of the uniqueness theorem of limit cycles, we define:

$$
\begin{aligned}
& \bar{\Omega}=\Gamma^{*} U \text { int } \Gamma^{*}, \Gamma^{*} \text { is the Poincare-Bendixson's outer boundary, } \\
& \Omega=\{(x, y) \mid(x, y) \in \bar{\Omega}, x>0\}, \\
& \Omega_{j}=\left\{(x, y) \mid(x, y) \epsilon \bar{\Omega}, \quad \operatorname{sgn}\left(\psi(x)+\xi(y)=(-1)^{\prime}\right\}, j=1,2,\right. \\
& \ell_{0}=\bar{\Omega} \cap \ell_{1},
\end{aligned}
$$

and let

$$
K(x, y)=\frac{H(x, y)}{\psi(x)+\xi(y)}, \quad(x, y) \in \Omega_{1} \cup \Omega_{2},
$$




$$
W(x, y)=\left(\phi^{\prime}(x) F^{\prime}(x)+\phi(x) F^{\prime \prime}(x)\right)(\xi(y)+\psi(x))-\psi^{\prime}(x) H(x, y),(x, y) \quad \epsilon \Omega,
$$

where $H(x, y)$ is defined as in (3.1).

Assume

$\left(\mathrm{H}_{5}\right):\left.H(x, y)\right|_{\ell_{0}}>0$ and $\left.W(x, y)\right|_{\Omega} \leq 0$, and

$\xi^{\prime}(y) \equiv 0$ or $\xi^{\prime}(y) \neq 0$ a.e. on $\ell_{0}$.

Since $W\left(x^{*}, y^{*}\right)<0$ implies $H\left(x^{*}, y^{*}\right)>0$, Theorem 3.4 guarantees that system $(2.1)$ has limit cycles in $\Omega$. Suppose $C_{1}$ and $C_{2}$ are two limit cycles around $\left(x^{*}, y^{*}\right)$ such that $C_{1} \subset C_{2}$ and, without loss of generality, suppose $C_{1}$ is stable from inside.

Let $Q$ be the point on $C_{1}$ such that

$$
x_{Q}=\min \left\{x \mid(x, y) \in C_{1}\right\}
$$

Since $\pi(y)$ is strictly increasing, $Q$ is unique. We claim that

LEMMA 3.5

$$
x_{Q} \leq \bar{x}
$$

PROOF. If $\xi^{\prime}(y) \equiv 0$ (or $\xi(y) \equiv 0$ ) on $\ell_{0}$, then (3.9) is clearly true. If $\xi^{\prime}(y) \neq 0$. Suppose (see Figure 3 ),

$$
x_{Q}>\bar{x} .
$$

Let $Q_{1}=\left(x_{Q_{1}}, y_{Q_{1}}\right)$ be the intersection of the ray $x=x_{Q}, y>0$ with $\ell_{0}$. Since $y_{Q_{1}}>0$ and (3.8), there exists $Q_{2}=\left(x_{Q_{2}}, y_{Q_{2}}\right)$ on $\ell_{0}$ such that $\bar{x}<x_{Q_{2}}<x_{Q}, 0<y_{Q_{2}}<y_{Q_{1}}$.

Let

$$
\epsilon_{1}=\min \left\{\frac{X_{Q}-X_{Q_{2}}}{2}, \frac{X_{Q_{2}}-\bar{x}}{2}, \frac{Y_{Q_{2}}}{2}\right\}
$$

Define an auxiliary function

$$
L(x, y)=H(x, y)-K\left(x_{Q}, y\right)(\psi(x)+\xi(y)),
$$

or, in the other form,

$$
L(x, y)=(\psi(x)+\xi(y))\left(K(x, y)-K\left(x_{Q}, y\right)\right)
$$

By (3.7),

$$
\frac{\partial K(x, y)}{\partial x} \leq 0
$$

We have

$$
L(x, y) \leq 0 \text { for }(x, y) \epsilon \Omega_{1} \cap\left\{(x, y) \mid 0<x<x_{Q}\right\} .
$$

On the other hand, $L(x, y)$ is continuous in the $\epsilon_{1}$-neighborhood of $Q_{2}: N_{\epsilon_{1}}\left(Q_{2}\right)$, and

$$
N_{\epsilon_{1}}\left(Q_{2}\right) \cap\left\{(x, y) \mid x=x_{Q}\right\}=\phi \text {. }
$$

Thus,

$$
\begin{aligned}
\left.L(x, y)\right|_{Q_{2}} & =H\left(x_{Q_{2}}, y_{Q_{2}}\right)-\frac{H\left(x_{Q}, y_{Q_{2}}\right)}{\psi\left(x_{Q}\right)+\xi\left(y_{Q_{2}}\right)}\left(\psi\left(x_{Q_{2}}\right)+\xi\left(y_{Q_{2}}\right)\right) \\
& =H\left(x_{Q_{2}}, y_{Q_{2}}\right)>0 .
\end{aligned}
$$

Hence, there exists $\epsilon \in\left(0, \epsilon_{1}\right)$ such that

$$
L(x, y)>0 \text { for }(x, y) \epsilon N_{\epsilon}\left(Q_{2}\right) \subset \Omega_{1} \cap\left\{(x, y) \mid \bar{x}<x<x_{Q}\right\},
$$

which is a contradiction to (3.13).

Therefore, the claim (3.9) holds. 
We also claim that on the periodic orbits $C_{i}$, we have

LEMMA 3.6

$$
\begin{aligned}
& \oint_{c_{i}} \operatorname{div}(\phi(x)(F(x)-\pi(y), \varrho(y)(\psi(x)+\xi(y)) d t \\
= & \oint_{C_{i}} L(x, y) d t, \text { for } i=1,2 .
\end{aligned}
$$

PROOF: By the Green formula,

$$
\begin{aligned}
& \oint_{c_{i}} \operatorname{div}(\phi(x)(F(x)-\pi(y), \varrho(y)(\psi(x)+\xi(y)) d t \\
= & \left.\oint_{c_{i}} \phi^{\prime}(x)(F(x)-\pi(y))+\phi(x) F^{\prime}(x)+\varrho^{\prime}(y)(\psi(x)+\xi(y))+\varrho(y) \xi^{\prime}(y)\right) d t \\
= & \oint_{c_{i}}(\psi(x)+\xi(y))\left(K(x, y)-\frac{1}{\psi(x)+\xi(y)}\left(\phi^{\prime}(x)(\pi(y)-F(x))\right.\right. \\
= & \oint_{c_{i}}(\psi(x)+\xi(y))\left(K(x, y)-K\left(x_{Q}, y\right) d t\right. \\
= & \oint_{C_{i}} L(x, y) d t, \quad i=1,2,
\end{aligned}
$$

since,

$$
\begin{aligned}
& \oint_{c_{i}}(\psi(x)+\xi(y))\left(K\left(x_{Q}, y\right)-\frac{1}{\psi(x)+\xi(y)}\left(\phi^{\prime}(x)(\pi(y)-F(x))\right.\right. \\
& =\oint_{c_{i}} \frac{K\left(x_{Q}, y\right)+\varrho^{\prime}(y)}{\varrho(y)} d y-\frac{\phi^{\prime}(x)}{\phi(x)} d x \\
& =\iint_{s_{i}}\left(\frac{\partial}{\partial x}\left(\frac{K\left(x_{Q}, y\right)+\varrho^{\prime}(y)}{\varrho(y)}\right)+\frac{\partial}{\partial y}\left(\frac{\phi^{\prime}(x)}{\phi(x)}\right)\right) d x d y \\
& =0 .
\end{aligned}
$$

Now, we are in the position to prove the following uniqueness theorem.

THEOREM 3.7. In addition to assumption $\left(\mathrm{H}_{5}\right)$, if

$$
(F(x)-\pi(y)) L_{y}(x, y) \geq 0 \text { for }(x, y) \epsilon \Omega,
$$

then there exists at most one limit cycle in system (2.1).

PROOF. As in Fig. 4, let $\ell_{0}$ intersect $C_{1}$ at $A_{1}, A_{2}, C_{2}$ at $B_{1}, B_{2}$.

Then

$$
\begin{aligned}
& C_{1}=\overparen{A_{1} A_{4}} \cup \overparen{A_{4} Q} \cup \overparen{Q A_{1}} \\
& C_{2}=\overparen{B_{3} B_{2}} \cup \overparen{B_{2} B_{4}} \cup \overparen{B_{4} B_{5}} \cup{\overparen{B_{5} B_{3}}}_{.}
\end{aligned}
$$


562

X. C. HUANG

Hence,

$$
\begin{aligned}
& \oint_{C_{1}} L(x, y) d t-\oint_{C_{2}} L(x, y) d t \\
& =\left[\left({\widetilde{A_{1} A_{4}}}-\int_{B_{3} B_{2}}\right)+\left(\int \widetilde{A_{4} Q}-\int_{B_{2} B_{4}}\right)+\left({\widetilde{Q A_{1}}}_{-\int_{B_{5} B_{3}}}\right)\right. \\
& \left.\quad-\int_{B_{4} B_{5}}\right] L(x, y) d t .
\end{aligned}
$$

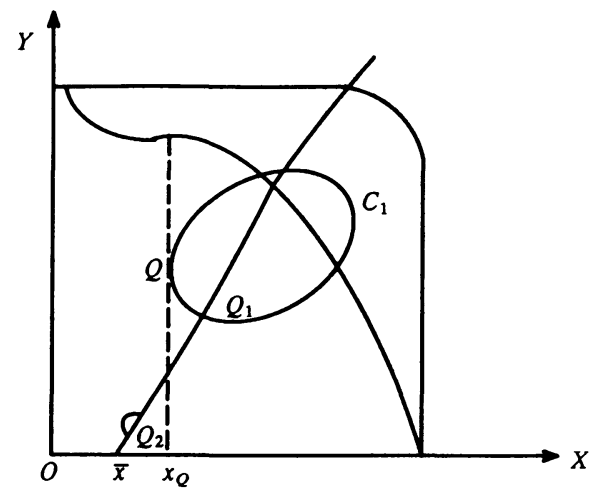

Fig. 3 The assumption $x_{Q}>\bar{x}$ results in a contradiction.

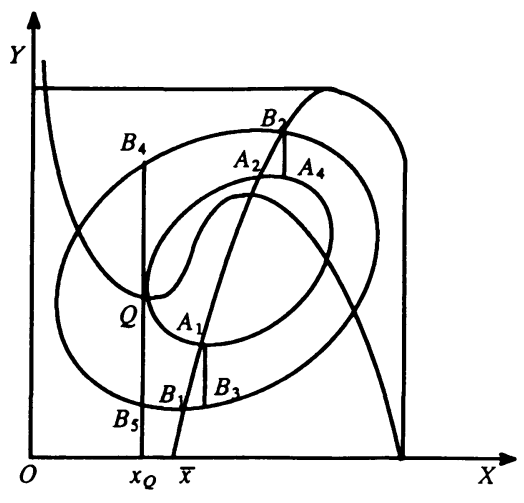

Fig. 4 It is impossible that the system (2.1) has two limit cycles.

It is not hard to see that

$$
\begin{aligned}
& \left(\int_{A_{1} A_{4}}-\int_{B_{3} B_{2}}\right) L(x, y) d t \\
& =-\oint_{B_{3} B_{2} A_{4} A_{1} B_{3}} L(x, y) d t+\left(\int_{B_{2} A_{4}}+\int_{A_{1} B_{3}}\right) L(x, y) d t \\
& =-\iint_{B_{3} B_{2} A_{4} A_{1} B_{3}} \frac{K_{x}(x, y)}{\varrho(y)} d x d y+\int_{B_{2} A_{4}} \frac{K(x, y)-K\left(x_{Q}, y\right)}{\varrho(y)} d y \\
& \quad+\int_{A_{1} B_{3}} \frac{K(x, y)-K\left(x_{Q}, y\right)}{\varrho(y)} d y \\
& >0,
\end{aligned}
$$

since $K_{x}(\mathrm{x}, \mathrm{y}) \leq 0$ and $K_{x}(\mathrm{x}, \mathrm{y}) \neq 0$ for $x_{\Lambda_{1}} \leq \mathrm{x}<\mathrm{K}$.

Suppose

$$
\widehat{A_{4} Q}: y=y_{1}(x), \quad x_{Q} \leq x \leq x_{A_{4}},
$$

and 


$$
\widetilde{B_{2} B_{4}}: y=y_{2}(x), \quad x_{Q} \leq x \leq x_{A_{4}}
$$

Then

$$
\begin{aligned}
& \left.\iint_{A_{4} Q}-\int_{B_{2} B_{4}}\right) L(x, y) d t \\
& =\int_{x_{Q}}^{x_{A_{4}}} \frac{L\left(x, y_{2}\right)}{\phi(x)\left(F(x)-\pi\left(y_{2}\right)\right)} d x-\int_{x_{Q}}^{x_{A_{4}}} \frac{L\left(x, y_{1}\right)}{\phi(x)\left(F(x)-\pi\left(y_{1}\right)\right)} d x \\
& =\int_{x_{Q}}^{x_{A_{4}}} \frac{\left(F(x)-\pi\left(y_{2}\right)\right)\left(L\left(x, y_{2}\right)-L\left(x, y_{1}\right)\right)+L\left(x, y_{2}\right)\left(\pi\left(y_{2}\right)-\pi\left(y_{1}\right)\right)}{\phi(x)\left(F(x)-\pi\left(y_{2}\right)\right)\left(F(x)-\pi\left(y_{1}\right)\right)} d x \\
& >0,
\end{aligned}
$$

since (3.17) and $\left(\psi(x)+\xi\left(y_{2}\right)\right)\left(K\left(x, y_{2}\right)-K\left(x_{Q}, y_{2}\right)\right) \geq 0$ for $x_{Q} \leq x \leq x_{A_{4}}$.

Similarly,

$$
\left(\int_{Q A_{1}}-\int_{B_{5} B_{3}}\right) L(x, y) d t \geq 0 .
$$

Finally,

$$
\begin{aligned}
& \int_{B_{4} B_{5}} L(x, y) d t=\int_{B_{4} B_{5}}(\psi(x)+\xi(y))\left(K(x, y)-K\left(x_{Q}, y\right)\right) d t \\
& =\int_{B_{4} B_{5}} \frac{\left(K(x, y)-K\left(x_{Q}, y\right)\right.}{\varrho(y)} d y \\
& =-\int_{y_{B_{5}}}^{y_{B_{4}}} \frac{K(x, y)-K\left(x_{Q}, y\right)}{Q(y)} d y \\
& \leq 0,
\end{aligned}
$$

since for $x<x_{Q}, K(x, y)-K\left(x_{Q}, y\right) \geq 0$.

From (3.19) to (3.22), we have

$$
\oint_{C_{1}} L(x, y) d t-\oint_{C_{2}} L(x, y) d t>0
$$

Since $\oint_{C_{1}} L(x, y) d t \leq 0$, so $\oint_{C_{2}} L(x, y) d t<0$.

Now, if we can prove $C_{1}$ is not a semi-stable limit cycle, then $C_{2}$ must be internally unstable. That is

$$
\oint_{C_{2}} L(x, y) d t \geq 0,
$$

which contradicts the fact

$$
\oint_{C_{2}} L(x, y) d t<0
$$

Now consider the following system containing a parameter $\gamma$ :

$$
\begin{aligned}
& \frac{d x}{d t}=\phi(x)(\bar{F}(x)-\bar{\pi}(y))=\bar{P} \\
& \frac{d y}{d t}=\varrho(y)(\psi(x)+\xi(y))=Q,
\end{aligned}
$$


where

$$
\begin{aligned}
& F(x)=F(x)-\gamma \psi(x) \\
& \bar{\pi}(y)=\pi(y)+\gamma \xi(y) .
\end{aligned}
$$

Let

$$
\begin{aligned}
& H(x, y)=\phi(x) \bar{F}^{\prime}(x)+\varrho(y) \xi^{\prime}(y) \\
& R(x, y)=\frac{\bar{H}(x, y)}{\psi(x)+\xi(y)}, \quad(x, y) \epsilon \Omega_{1} \cup \Omega_{2}
\end{aligned}
$$

and

$$
W(x, y)=\left(\phi^{\prime}(x) \bar{F}^{\prime}(x)+\phi(x) \bar{F}^{\prime \prime}(x)\right)(\xi(y)+\psi(x))-\psi^{\prime}(x) H(x, y) .
$$

Clearly, if $\gamma$ is small enough, then all the assumptions for system (2.1) are satisfied for system (3.24). Thus, if system (3.24) has two limit cycles $\overline{C_{1}}$ and $\overline{C_{2}}, \overline{C_{1}} \subset \overline{C_{2}}$, then we have

$$
\oint_{\bar{C}_{2}} \operatorname{div}(\bar{P}, Q) d t<\oint_{\bar{C}_{1}} \operatorname{div}(\bar{P}, Q) d t \leq 0 .
$$

Furthermore, let

$$
\bar{\beta}(x, y, \gamma)=\tan ^{-1} \frac{Q(x, y, \gamma)}{\bar{P}(x, y, \gamma)}
$$

Then

$$
\begin{aligned}
\frac{\partial \bar{\beta}}{\partial \gamma} & =\frac{\frac{\partial Q}{\partial \gamma} \bar{P}-Q \frac{\partial \bar{P}}{\partial \gamma}}{\bar{P}^{2}+Q^{2}} \\
& =\frac{Q(y) \phi(x)(\psi(x)+\xi(y))^{2}}{\bar{P}^{2}+Q^{2}} \geq 0
\end{aligned}
$$

for all ordinary points $(x, y)$ of system (3.24).

Also, the equilibrium points of system (3.24) are not dependent on $\gamma$. Thus, system (3.24) forms a generalized rotated vector field in $\Omega$.

According to the theory of generalized rotated vector field (see [9], for example), for sufficiently small $\gamma>$ 0 , system (3.24) produces a generalized limit cycle $\overline{C_{1}} \subset C_{1}$ which is at least stable internally and a generalized limit cycle $\overline{C_{2}} \supset C_{1}$ which is at least unstable on one side. This is a contradiction to (3.25). We, thus, complete the proof of Theorem 3.7 .

4. EXAMPLES AND DISCUSSION.

EXAMPLE 1. (Huang 1988 [5], Huang and Merrill, 1989 [6])

$$
\begin{aligned}
& \frac{d x}{d t}=\phi(x)(F(x)-\pi(y)) \\
& \frac{d y}{d t}=\varrho(y) \psi(x)
\end{aligned}
$$

with the assumptions $\left(\mathrm{H}_{1}\right)-\left(\mathrm{H}_{4}\right)$ in [6].

It is easy to see that system $(4.1)$ is a special case that $\xi(y) \equiv 0$ in $(2.1)$; and the assumptions $\left(\mathrm{H}_{1}\right)-\left(\mathrm{H}_{4}\right)$ in Chapter 2 of this paper are satisfied. Therefore, Theorem 3.1 implies that if $F^{\prime}\left(x^{*}\right)>0$ the equilibrium point $\left(x^{*}, y^{*}\right)$ of $(4.1)$ is unstable, and if $F^{\prime}\left(x^{*}\right)<0$ it is stable. Theorem 3.4 tells us that when $\left(x^{*}, y^{*}\right)$ is unstable there exists at least one limit cycle in (4.1). For the uniqueness of limit cycles, by Theorem 3.7, we have:

THEOREM 4.1. (Huang and Merrill [6]) In addition to the assumptions $\left(\mathrm{H}_{1}\right)-\left(\mathrm{H}_{4}\right)$ in [6], if

$$
F^{\prime}\left(x^{*}\right)>0 \text { and }\left(\frac{\phi(x) F^{\prime}(x)}{\psi(x)}\right)^{\prime} \leq 0,
$$

then there is a unique limit cycle around the equilibrium point $\left(x^{*}, y^{*}\right)$ in $(4.1)$. 
PROOF. Since $\xi(y)=0$,

$$
F^{\prime}\left(x^{*}\right)>0 \text { and }\left(\frac{\phi(x) F^{\prime}(x)}{\psi(x)}\right), \leq 0
$$

$\left(\mathrm{H}_{5}\right)$ is hold. Furthermore, if $\xi(y)=0$,

$$
L(x, y)=\phi(x) F^{\prime}(x)-\frac{\phi(x) F^{\prime}(x)}{\psi\left(x_{Q}\right)} \psi(x)
$$

which is only a function of $x$. Hence $L_{y}(x, y)=0$, and consequently $(F(x)-\pi(y)) L_{y}(x, y)=0$ for $(x, y) \in \Omega$. Therefore, the conditions of Theorem 3.7 are all satisfied. Employing theorem 3.7 will end the proof of Theorem 4.1 .

REMARK. In [6], when we proved the uniqueness of limit cycles in (4.1), we employed the Zhang theorem (or equivalent, Cherkas and Zhilevich theorem). But in this paper we do not need to use it. We also may simplify our assumptions for the existence and uniqueness of limit cycles because some of the assumptions are made for the global stability such as Theorems 3.2 and 3.3.

The Zhang theorem and Cherkas and Zhilevich theorem can be found in [9].

EXAMPLE 2. (Kuang and Freedman, 1988 [7])

$$
\begin{aligned}
& \frac{d x}{d t}=x g(x)-\xi(y) p(x) \\
& \frac{d y}{d t}=\eta(y)(-\gamma+q(x))
\end{aligned}
$$

with the assumptions $\left(\mathrm{H}_{1}\right)-\left(\mathrm{H}_{8}\right)$ in [7].

Clearly, system (4.3) is a special case of (4.1) and hence if all the assumptions in this paper are satisfied, Theorem 3.7 is applicable.

The original proof of the uniqueness of limit cycles in [7] is based on Zhang's theorem. However, since the assumptions $\left(\mathrm{H}_{1}^{*}\right),\left(\mathrm{H}_{2}\right)$ and $\left(\mathrm{H}_{4}\right)$ in this paper are not assumed there, the existence of limit cycles is not guaranteed and some arguments need to be modified.

As an example, let us consider the following system:

$$
\begin{aligned}
& \frac{d x}{d t}=x\left(1+2 x-x^{2}\right)-y x^{\frac{3}{2}} \\
& \frac{d y}{d t}=y\left(-\gamma+x^{\frac{3}{2}}\right),
\end{aligned}
$$

which satisfies all the required hypotheses by Kuang and Freedman [7]. Unfortunately, since (4.4) in [7],

$$
\begin{aligned}
u & =\int_{0}^{x} \frac{d s}{P\left(s+x^{*}\right)}=\int_{0}^{x} \frac{d s}{\left(s+x^{*}\right)^{\frac{3}{2}}} \\
& =\frac{2}{\sqrt{x^{*}}}-\frac{2}{\sqrt{X+x^{*}}} .
\end{aligned}
$$

By (4.1) in [7], $X+x^{*}=x$. Hence

$$
u \rightarrow \begin{cases}\frac{2}{\sqrt{x^{*}}} & \text { when } X \rightarrow+\infty \\ -\infty & \text { when } x \rightarrow-x^{*}\end{cases}
$$

Therefore, $u$ has no definition on

$$
\left(\frac{2}{\sqrt{x^{*}}},+\infty\right)
$$


and consequently the hypothesis that $\phi(v)+F(u)=0$ is defined for all $u \epsilon(-\infty,+\infty)$ has not been satisfied. Thus, Zhang's theorem is not applicable and the uniqueness of limit cycles can not be obtained by the argument in [7].

Clearly, system (4.4) does satisfy all the requirements of Theorem 4.1. So if our theorem is employed we can still have the uniqueness result for system (4.3).

The idea used in this paper is possible for use in determining the uniqueness conditions of limit cycles in the general Kolmogorov system. This work will be published in a separate paper.

ACKNOWLEDGMENT. The author extends his thanks to Professor Stephen J. Merrill for his valuable discussions.

\section{REFERENCES}

1. ALBRECHT, F., GATZKE, H. and WAX, N. Stable limit cycles in prey-predator populations, Science 181 (1973), 1073-1074.

2. CHENG, K.S. Uniqueness of a limit cycle for a predator-prey system, SLAM J. Math. Anal. 12 (1981), 541-548.

3. HARRISON, G.W. Global stability of predator-prey interaction, L. Math. Biol. 8 (1979), 159-171.

4. HARRISON, G.W. Bull. Math. Biol. 48 (1986), 137-148.

5. HUANG, X.C. Uniqueness of limit cycles of generalized Lienard systems and predator-prey systems, L. Phys. A. Math. Gen. 21 (1988), L685-691.

6. HUANG, X.C. and MERRILL, S.J. Conditions for uniqueness of limit cycles of general predator-prey systems, Math. Biosci. (1989), To appear.

7. KUANG, Y. and FREEDMAN, H.I. Uniqueness of limit cycles in Gause-type models of Predator-prey systems, Math. Biosci. 88 (1988), 67-84.

8. MAY, R.M. Limit cycles in predator-prey communities, Science 177 (1972), 900-902.

9. YE, Y.Q., et al. Theory of Limit Cycles, Amer. Math. Soc., Providence, R.I., 1986. 


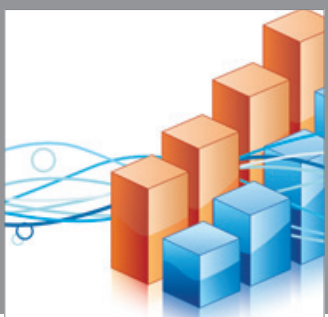

Advances in

Operations Research

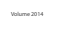

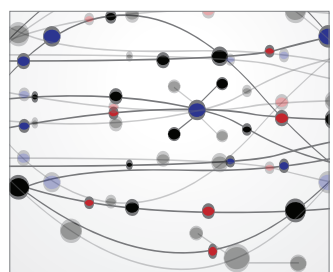

\section{The Scientific} World Journal
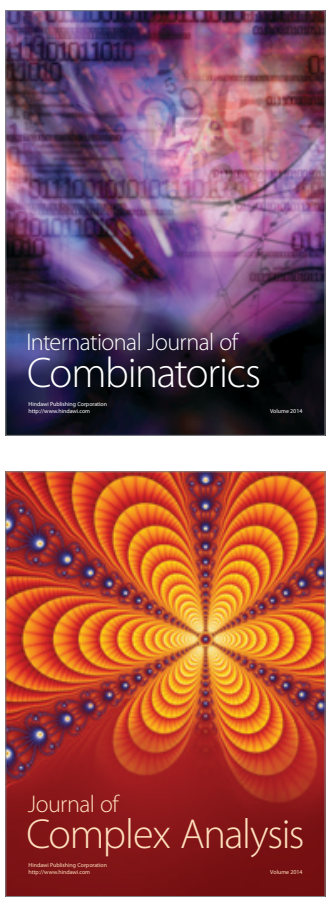

International Journal of

Mathematics and

Mathematical

Sciences
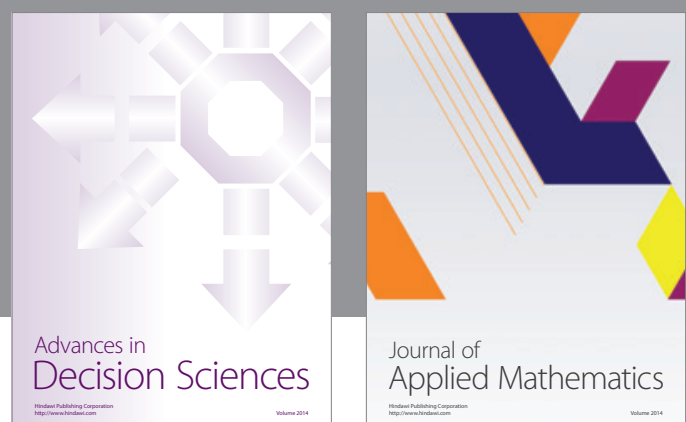

Journal of

Applied Mathematics
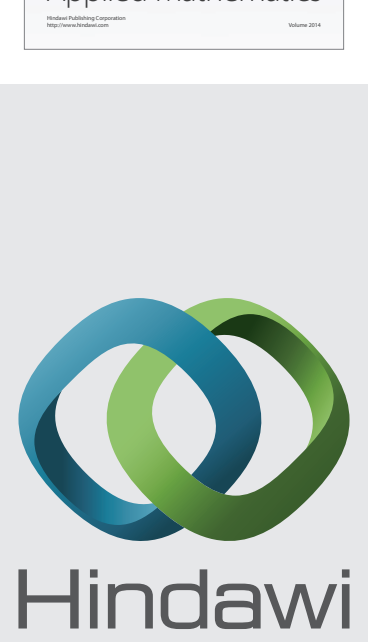

Submit your manuscripts at http://www.hindawi.com
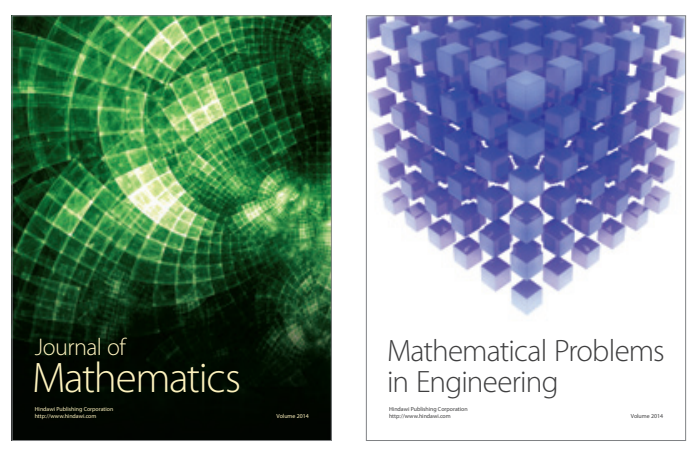

Mathematical Problems in Engineering
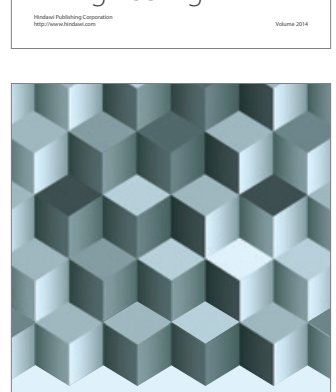

Journal of

Function Spaces
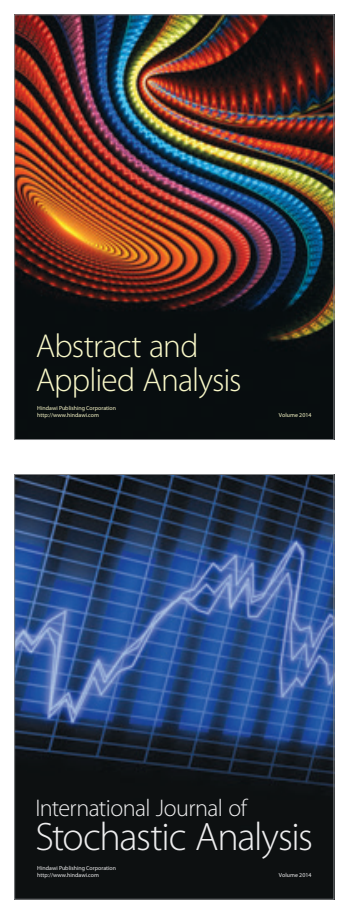

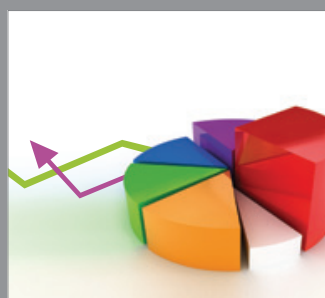

ournal of

Probability and Statistics

Promensencen
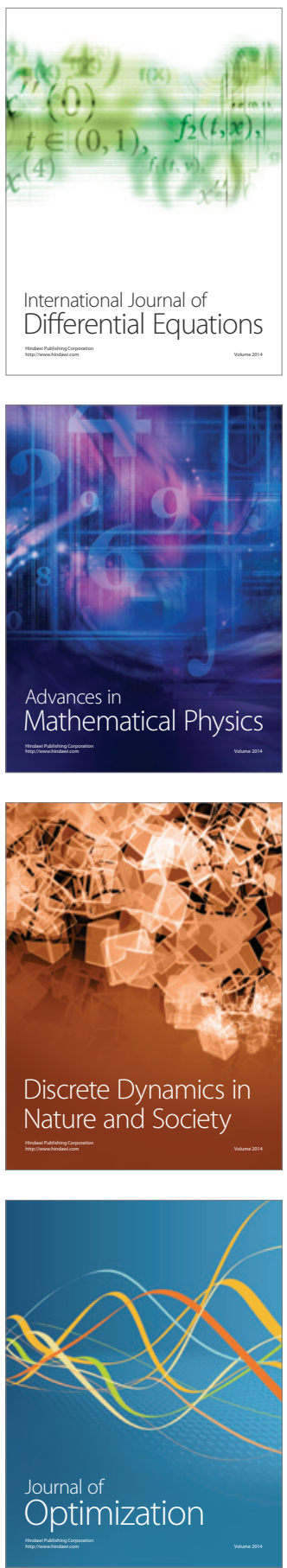